\title{
Early findings of a novel established molecular diagnostic technique for the prediction of malignant transformation in leukoplakia
}

\author{
JUTTA RIES $^{1}$, SABINE PONADER ${ }^{1}$, NUR MOLLAOGLU ${ }^{1,2}$, ELEFTHERIOS VAIRAKTARIS $^{3}$, \\ FRIEDRICH W. NEUKAM ${ }^{1}$ and EMEKA NKENKE ${ }^{1}$ \\ ${ }^{1}$ Department of Oral and Maxillofacial Surgery, University of Erlangen, Erlangen, Germany; \\ ${ }^{2}$ Department of Oral and Maxillofacial Surgery, Gazi University, School of Dentistry, Ankara, Turkey; \\ ${ }^{3}$ Department of Oral and Maxillofacial Surgery, University of Athens Medical School, Athens, Greece
}

Received June 9, 2009; Accepted July 16, 2009

DOI: $10.3892 / \mathrm{mmr} 00000197$

\begin{abstract}
To date, there are no objective parameters regarding the early prediction of malignant transformation in leukoplakia. Expression analysis of melanoma-associated antigens (MAGE-A) can differentiate between healthy and already malignant transformed tissues. Thus, expression analysis may also be used as an additional diagnostic tool for oral pre-malignant lesions to monitor potential malignant changes. In this study, four specimens collected from the same patient within a year were examined. Specimens were taken from the part of the lesion that displayed a rapid progression from fibroma to oral squamous cell carcinoma (OSCC). Clinically and histopathologically, the oral lesion was first diagnosed as fibroma with inflammatory infiltration, then as leukoplakia with hyperplasia, then as leukoplakia with severe dysplasia, and lastly as OSCC. Expression of MAGE-A1, -A3, -A4, -A6, -A10 and -A12 was investigated in the frozen tissue specimens using RT-PCR and quantitative real-time RT-PCR. There was no expression of MAGE-A in the specimen of fibroma with inflammatory infiltration. However, four genes were expressed by the second specimen of leukoplakia with hyperplasia. With the exception of MAGE-A1, all antigens were expressed in the specimens, which were histopathologically diagnosed as leukoplakia with severe dysplasia and OSCC. Expression analysis of six different MAGE-A genes indicated a high potential for malignant change in the specimens diagnosed as leukoplakia that eventually developed into OSCC. Thus, analysis of MAGE-A expression can predict malignant transformation in leukoplakia.
\end{abstract}

Correspondence to: Dr Jutta Ries, Department of Oral and Maxillofacial Surgery, University of Erlangen, Glueckstrasse 11, 91054 Erlangen, Germany

E-mail: jutta.ries@uk-erlangen.de

Key words: melanoma-associated antigen expression, real-time reverse transcriptase-polymerase chain reaction, oral pre-cancerous lesions, diagnosis, oral squamous cell carcinoma

\section{Introduction}

The poor prognosis of patients who suffer from advanced oral squamous cell carcinoma (OSCC) is in large part due to the absence of sensitive and specific markers for the early detection of primary tumors or malignant cells that may be used to determine the risk of tumor development. Primary tumors may originate from a pre-cancerous oral lesion such as leukoplakia, erythroplakia or lichen planus (1). The early identification of oral lesions with a high risk of malignant transformation as well as the early detection of oral tumors remain important clinical issues to be solved. Leukoplakia is the most frequent progenitor of OSCC. It has been reported that between 11 and $67 \%$ of all OSCCs develop from such pre-malignant lesions (2).

Oral carcinogenesis is a multistep process involving a series of molecular changes that occur before clinical or histopathological evidence of malignancy. Thus, early molecular changes act as important markers for the early diagnosis of OSCC and the cancer risk assessment of oral pre-cancerous lesions. Melanoma-associated antigens (MAGE)-A are not expressed in normal adult tissues, with the exception of male germ cells, but are often expressed in a variety of tumor tissues (3-7). For example, MAGE-A is also expressed in a high percentage of oral tumors, but not in healthy normal oral mucosa (NOM) (8-10). Due to their high specificity to oral cancer cells, MAGE-A are suitable markers for use in various diagnostic applications. However, tumor detection using a single antigen is not adequately sensitive for diagnostic applications due to a low expression frequency and heterogeneous expression pattern. In previous studies, expression frequency was found to be higher when multiple MAGE-A were applied (9-12). Thus, multiple expression analysis using several MAGE-A may allow a distinction between healthy and already malignant transformed tissues, even in the early stages of OSCC, with a very high accuracy rate. In addition, analysis of MAGE-A expression by reverse transcriptase-polymerase chain reaction (RT-PCR) may be a useful tool for detecting small numbers of neoplastic cells, which might be microscopically undetectable, to predict the cancer risk assessment of oral pre-malignant lesions that may transform into OSCC over time. 
Table I. Histopathological results and MAGE-A expression analysis of normal oral mucosa, fibroma with inflammatory infiltrations, hyperplastic leukoplakia, severe dysplastic leukoplakia, and arising squamous cell carcinoma (pT1, pN0, Mx, G1).

\begin{tabular}{|c|c|c|c|c|c|c|c|c|}
\hline \multirow[b]{2}{*}{ Histopathological diagnosis } & \multirow[b]{2}{*}{ Date of biopsy } & \multirow[b]{2}{*}{ Region } & \multicolumn{6}{|c|}{ MAGE-A expression (\%) } \\
\hline & & & A1 & A3 & A4 & A6 & A10 & A12 \\
\hline $\begin{array}{l}\text { Normal oral mucosa } \\
\text { (-) control }\end{array}$ & & & - & - & - & - & - & - \\
\hline Inflamed fibroma & $10 / 1996$ & $31-48$ & - & - & - & - & - & - \\
\hline Hyperplastic LK & $3 / 1997$ & $31-48$ & - & + & + & + & - & + \\
\hline Severe dysplastic LK & $6 / 1997$ & $43-45$ & - & + & + & + & + & + \\
\hline $\begin{array}{l}\text { OSCC } \\
(\mathrm{pT} 1, \mathrm{pN} 0, \mathrm{Mx}, \mathrm{G} 1)\end{array}$ & 9/1997 & $43-48$ & - & + & + & + & + & + \\
\hline
\end{tabular}

LK, leukoplakia; OSCC, oral squamous cell carcinoma.

The aim of this study was to determine the expression of six different MAGE-A genes in a persistent pre-malignant leukoplakia lesion, to perform cancer risk assessment to predict the possible malignant transformation of the lesion over time, and to propose improved treatment planning for the patient.

\section{Materials and methods}

Tissues. The specimens investigated in this study were collected from the lesion of one patient, a 75-year-old Caucasian female who underwent close and regular clinical follow-up at the Department of Oral and Maxillofacial Surgery, University of Erlangen. The lesion was located in the right mandibular vestibulum area and displayed recurrent locoregional changes, which resulted in OSCC within a year. Clinically, the changes in the oral mucosa manifested as leukoplakia with four different histopathological stages (Table I). Each collected specimen was divided into two pieces. One piece was histologically examined and the second was immediately snap frozen and stored at $-80^{\circ} \mathrm{C}$ until molecular examination. Informed consent from the patients and the approval of the local ethics committee were obtained. After the excision of the lesion, the patient underwent close follow-up at regular intervals. As of January 2009, medical examination had revealed no cases of recurrence or secondary tumors.

A pooled sample of whole RNA isolated from $20 \mathrm{NOM}$ samples obtained from otherwise healthy volunteers and from testis were respectively included as negative and positive controls for the RT-PCR analysis.

RNA isolation. Whole RNA was isolated using a commercially available kit according to the manufacturer's instructions (RNeasy Mini Kit; Qiagen, Hilden, Germany). The quality of the RNA was assessed using the One Step RT-PCR Kit (Qiagen) with glyceraldehyde-3-phosphate-dehydrogenase (GAPDH)specific primers for cDNA amplification. GAPDH-PCR products were analyzed on a $2 \%$ agarose gel. To exclude false positive results generated by the amplification of genomic DNA sequences that were not totally eliminated by DNA digestion, purified
$\mathbf{A}$

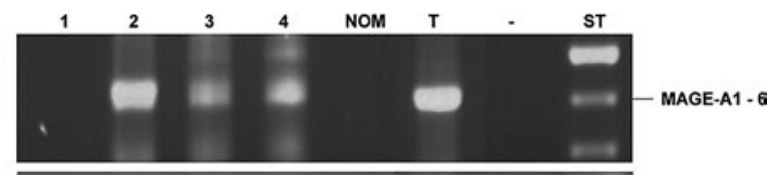

B

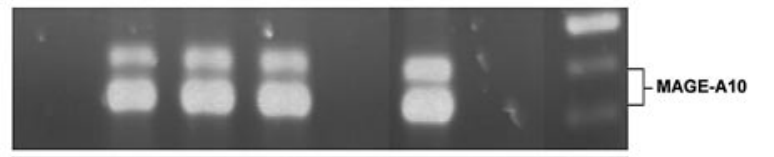

C

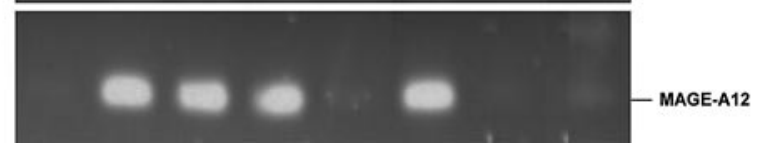

Figure 1. Analysis of MAGE-A expression in leukoplakia, oral squamous cell carcinoma (OSCC) and normal oral mucosa. (A) Simultaneous analysis of MAGE-A1 to -A6 in one RT-PCR reaction. (B) Detection of MAGE-A10. (C) MAGE-A12 expression. Lane 1, fibroma with inflammatory infiltration; 2, hyperplasia; 3, severe dysplasia; 4, OSCC. NOM, normal oral mucosa; T, testis as positive control; -, water as negative control; ST, standard..

RNA from each specimen was tested for the amplification of genomic GAPDH using specific primers for PCR. GAPDH-PCR products were analyzed on a $2 \%$ agarose gel. Only RNA isolations showing the specific amplification product of RT-PCR and no visible band for genomic amplification were analyzed in the subsequent procedures.

\section{RT-PCR and real-time RT-PCR methods.}

$R T-P C R$. MAGE-A1-6 assay was used for the simultaneous detection of MAGE-A1-A6 (10). The determination of the specific MAGE-A member expressed was carried out by real-time RT-PCR. For the analysis of MAGE-A12 expression, nested RT-PCR was used as described by Molloaglu et al (8). Analysis of MAGE-A10 was performed using the One Step RT-PCR Kit and MAGE-A10 specific primers. Cycling conditions for RT-PCR were as follows: reverse transcription at $50^{\circ} \mathrm{C}$ for $30 \mathrm{~min}$, initial PCR activation at $95^{\circ} \mathrm{C}$ for $5 \mathrm{~min}$, denaturation at $95^{\circ} \mathrm{C}$ for $30 \mathrm{sec}$, annealing at $60^{\circ} \mathrm{C}$ for $30 \mathrm{sec}$, and elongation at $72^{\circ} \mathrm{C}$ for $45 \mathrm{sec}$, with a final extension at $72^{\circ} \mathrm{C}$ for $10 \mathrm{~min}$. Gene specific primer sequences, cycle number 
Table II. Primers for the amplification of GAPDH and MAGE-A members.

\begin{tabular}{|c|c|c|c|c|}
\hline Primer & Primer sequence & Cycles & Size (bp) & Annealing $\left({ }^{\circ} \mathrm{C}\right)$ \\
\hline GAPDH (s) & GCAGGGGGGAGCCAAAAGGG & 35 & 567 & 60 \\
\hline GAPDH (as) & TGCCAGCCCCAGCGTCAAAG & & & \\
\hline \multicolumn{5}{|l|}{ MAGE-A1-6 Assay } \\
\hline MMRP1 (o) & CTGAAGGAGAAGATCTGCC & 30 & $831-855$ & 60 \\
\hline MMRP2 (o) & CTCCAGGTAGTTTTCCTGCAC & & & \\
\hline MMRP3 (i) & CTGAAGGAGAAGATCTGCCWGTG & 30 & $469-493$ & 60 \\
\hline MMRP4 (i) & 5-CCAGCATTTCTGCCTTTGTGA-3 & & & \\
\hline \multicolumn{5}{|l|}{ MAGE-A10 } \\
\hline A10 (s) & GGAACСССТСТTTTCTACAGAC & 40 & 411 & 60 \\
\hline A10 (as) & TCCTCTGGGGTGCTTGGTATTA & & 501 & \\
\hline \multicolumn{5}{|l|}{ RT-nPCR } \\
\hline MAGE-A12 (o,s) & TCCGTGAGGAGGCAAGGTTC & 30 & 341 & 60 \\
\hline MAGEA12 (o,as) & ATCGGATTGACTCCAGAGAGTA & & & \\
\hline MAGE-A12 (i,s) & TCCGTGAGGAGGCAAGGTTC & 30 & 181 & 58 \\
\hline MAGEA12 (i,as) & GAGCCTGCGCACCCACCAA & & & \\
\hline
\end{tabular}

aDetection of two transcript variants due to different splicing. s, sense; as, antisense; MMRP, multiple MAGE-A1-A6 recognizing primer. o, outer primer of nested RT-PCR; i, inner primer of nested RT-PCR.

annealing temperature and the expected sizes of the amplified fragments are shown in Table II. PCR products were separated on $1 \%$ agarose gels and stained with ethidium bromide for the identification of the amplification products by size (Fig. 1).

Real-time RT-PCR. Reverse transcription from total RNA was carried out using the Transcriptor High Fidelity cDNA Synthesis Kit (Roche, Mannheim, Germany) according to the manufacturer's instructions.

The QuantiTect ${ }^{\mathrm{TM}}$ SYBR ${ }^{\circledR}$ green PCR Kit (Qiagen) and QuantiTect Primer Assays (Hs_MAGEA1_2SG QuantiTect Primer Assay for MAGE-A1, Hs_MAGEA3_1SG QuantiTect Primer Assay for MAGE-A3, Hs_MAGEA4_1SG QuantiTect Primer Assay for MAGE-A4, Hs_MAGEA6_1SG QuantiTect Primer Assay for MAGE-A6, Hs_MAGEA10_1SG QuantiTect Primer Assay for MAGE-A10 and Hs_MAGEA12_1SG QuantiTect Primer Assay for MAGE-A12 (Qiagen) were used for real-time PCR analysis according to the manufacturer's protocols. In brief, $50 \mathrm{ng}$ of cDNA was used for each PCR reaction in a total volume of $25 \mu \mathrm{l}$. The cycling conditions used for the real-time PCR were as follows: initial denaturation/ enzyme activation for $15 \mathrm{~min}$ at $95^{\circ} \mathrm{C}$, followed by 40 cycles of denaturation at $94^{\circ} \mathrm{C}$ for $15 \mathrm{sec}$, annealing at $55^{\circ} \mathrm{C}$ for $30 \mathrm{sec}$, and elongation at $72^{\circ} \mathrm{C}$ for $34 \mathrm{sec}$. The production of a single product was confirmed by melting curve analysis. A Ct value $<33$ was recommended as an indicator of the positive expression of the target gene. All reactions were run in triplicate and verified by a second analysis. Results are displayed in Fig. 2.

\section{Results}

Clinical and histopathological classification of the collected specimens. The lesion clinically occurred as white patches histopathologically classified as follows: first, as fibroma with inflammatory infiltration, then as leukoplakia with hyperplasia, then as severe dysplasia, and lastly as OSCC classified as pT1, pNO, Mx, G1 according to the TNM classification.

The first specimen was collected in November 1996. Clinical investigation revealed a white verrucous lesion with inflamed erosive areas in the right mandibular vestibulum area (between the teeth) (31-48). Histopathological examination revealed fibroma with inflammatory infiltration, which was not evaluated as pre-cancerous. Four months later, in March 1997, a diffuse white lesion manifested in the same region and was histopathologically diagnosed as leukoplakia with hyperplasia. In June 1997, histological investigation revealed that the persistent leukoplakia had progressed to a severe dysplasia, again localized in the same region. Finally, in September 1997, the lesion had been transformed into OSCC according to the TNM classification of pT1, pN0, Mx, G1 (Table I).

Expression of MAGE-A by the potential pre-malignant oral lesions. Molecular-based investigation revealed that the fibroma with inflammatory infiltration did not express any of the MAGE-A genes analyzed. However, MAGE-A3, -A4, -A6 and -A12 were expressed by the secondary developed locoregional hyperplastic leukoplakia. Furthermore, with the exception of MAGE-A1, all the examined antigens were expressed by the severe dysplastic leukoplakia and OSCC that subsequently appeared in the same area. Figs. 1 and 2 display the results of the amplification of MAGE-A3, -A4, -A6, -A10 and $-\mathrm{A} 12$ in the endpoint-RT-PCR and real-time RT-PCR analyses, respectively. The MAGE-A expression patterns of the four biopsy specimens taken from the same patient from the same area within a year are displayed in Table I. 


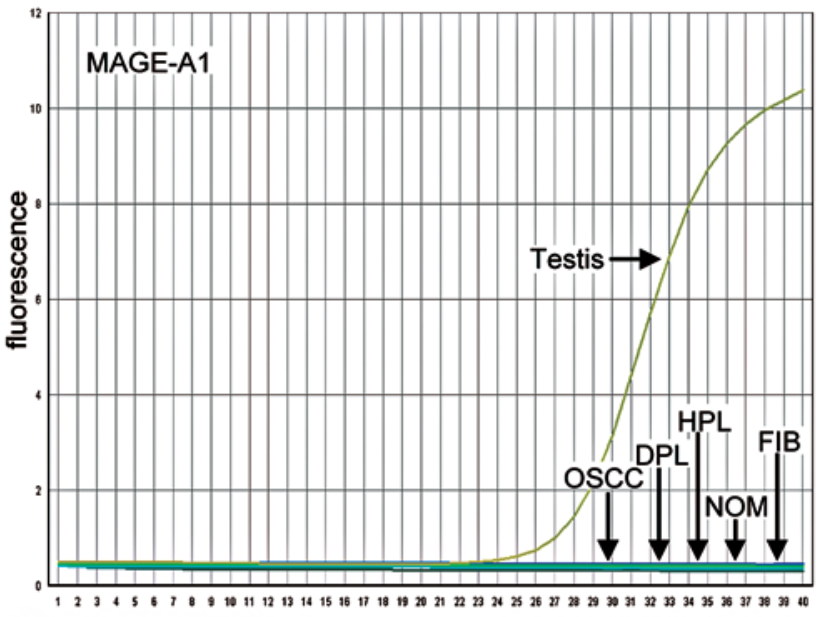

Cycle number

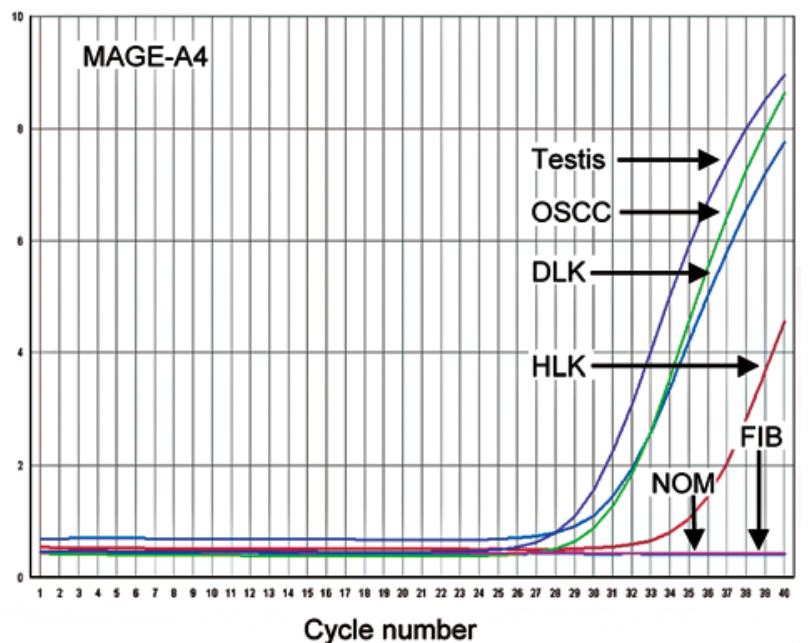

Cycle number

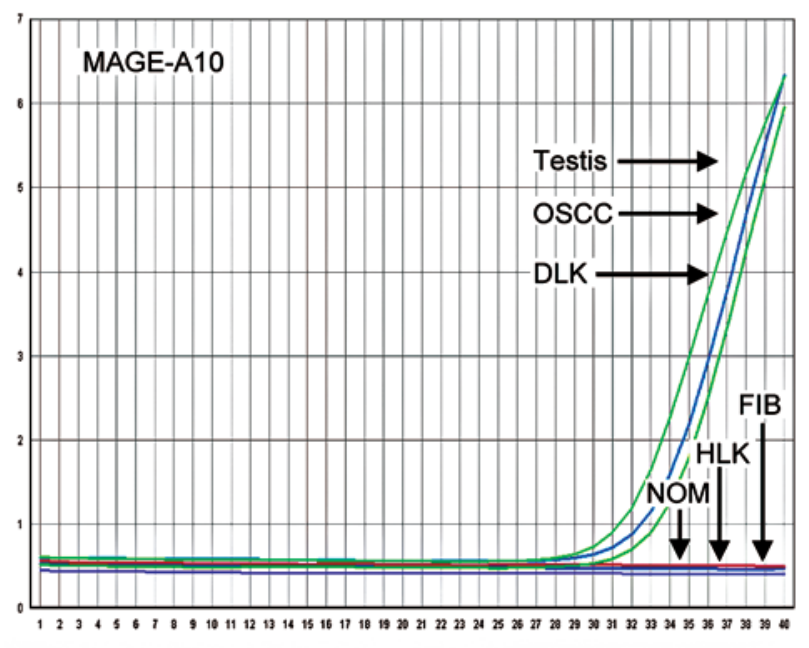

Cycle number

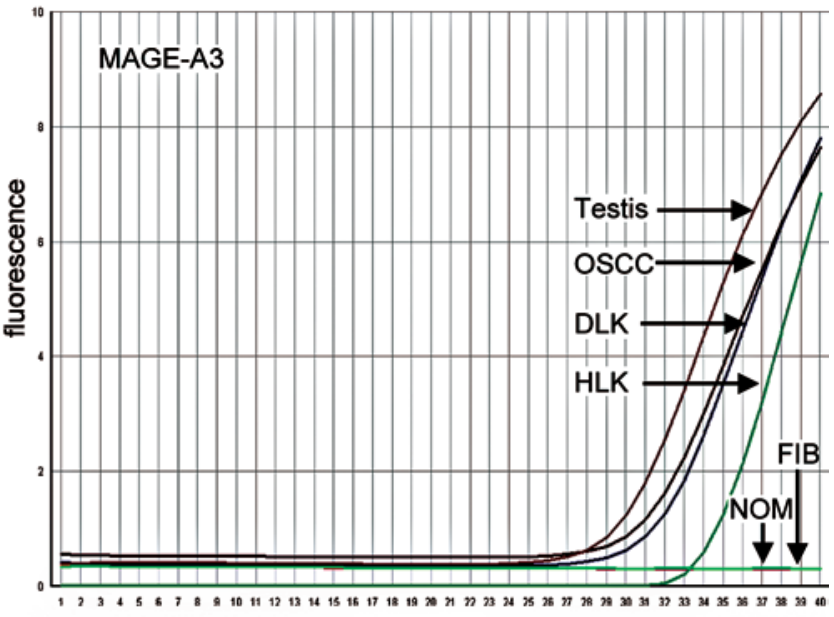

Cycle number

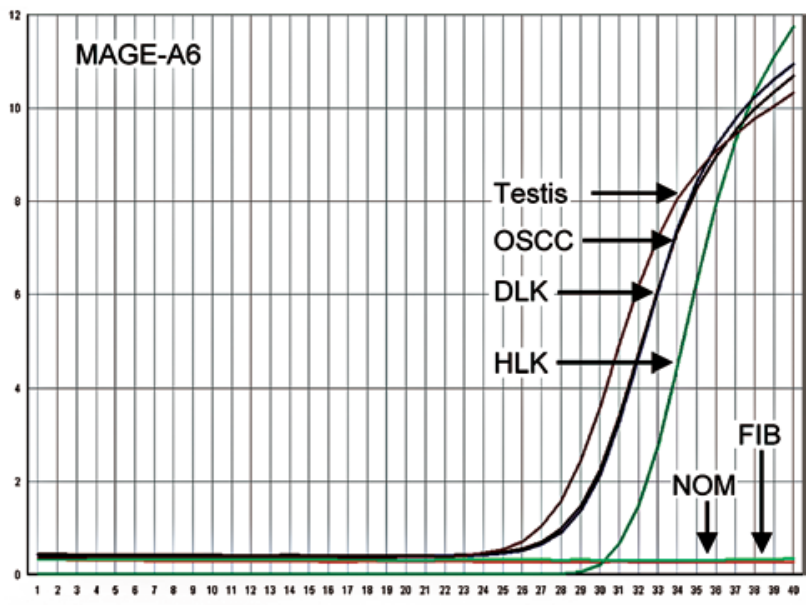

Cycle number

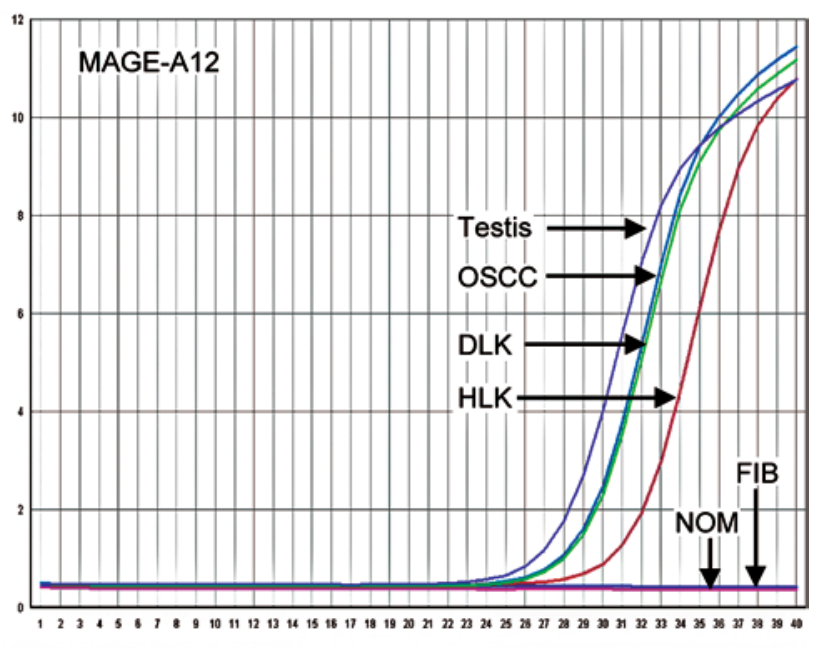

Cycle number

Figure 2: Expression analysis of MAGE-A1, -A3, -A4, -A6, -A10 and -A12 by real-time RT-PCR in fibroma (FIB), leukoplakia with hyperplasia (HLK) and dysplasia (DLK), oral squamous cell carcinoma (OSCC) and normal oral mucosa (NOM).

\section{Discussion}

Certain oral mucosal lesions, such as leukoplakia, lichen planus and erythroplakia, are known to have a high potential for malignant transformation. Between 1 and $18 \%$ of oral leukoplakia undergo malignant transformation, significantly increasing the incidence of oral cancer $(13,14)$. To date, the best predictor of the potential for malignant transformation 
is the histopathological examination of the lesions (15). Since the histological examination of potentially malignant lesions may not always be straightforward, and since the results may vary according to numerous subjective factors, the availability of molecular criteria would improve the prediction of cancer risk in certain oral lesions $(16,17)$. In such cases, the early molecular diagnosis of cell turnover may improve accuracy when the lesion being observed is considered to be clinically indefinite for a long period of time. It would also aid in the determination of when and where to biopsy. Thus, it is likely that patient survival rate and quality of life would considerably improve. In the present study, we detected the expression of various MAGE-A in leukoplakia, which is considered to be an oral pre-malignant lesion.

Oral carcinogenesis is driven by genetic changes, which often occur due to the altered expression patterns of several genes. It is postulated that certain genetic changes are accompanied by definitive histopathological appearances in oral carcinogenesis. In most cancer genomes, one of the hallmarks of molecular events associated with tumor progression is epigenetic modification. Epigenetic modification refers to changes in the global DNA methylation level of the genome, leading to changes in the transcription rate of several genes and, subsequently, to alterations in their expression pattern. In addition, it is now well established that such gene-specific hypomethylation and regional hypermethylation occur during the progression to tumorigenesis of OSCC (18-21). The precise regulatory mechanism of MAGE-A expression is not yet fully understood; however, it has been demonstrated to be linked to overall DNA demethylation, especially in the promoter regions (22-24). Therefore, it is accepted that the reactivation and transcription of these genes is an early step within oral carcinogenesis development, and may serve to pinpoint the malignant transformation. As a result, the analysis of MAGE-A expression in oral lesions may be useful as a considerably early diagnostic tool. Once the expression of a particular MAGE-A is de-repressed, its expression is retained during tumor progression, and accumulates during the progression of the malignancy.

It has been postulated that MAGE-A expression is restricted to malignant and germ cells. In this study, the expression of MAGE-A was investigated in biopsy specimens taken from a persistent oral lesion in the same patient at different time intervals within a year. Unlike previous findings, which determined the exclusive expression of MAGE-A in testis and tumor tissues, the present study demonstrated for the first time the expression of MAGE-A in potentially precancerous oral lesions, and revealed the expression rate of MAGE-A3, -A4, -A6 and -A12 by hyperplastic and dysplastic leukoplakia. A progressive histopathological change was observed in the lesion, ranging from fibroma to OSCC. Histopathologically, the first specimen was revealed to be fibrosis with inflammatory infiltration, which is not considered to be a precursor of malignancy. This was followed by leukoplakia with hyperplasia, and then by leukoplakia with severe dysplastic changes. Eventually, these developed into OSCC. Molecular-based investigations revealed that neither NOM nor inflamed fibrosis expressed MAGE-A. Expression of MAGE-A was determined only once the histopathological changes had progressed.
Hyperplastic leukoplakia is not considered a precursor for malignancy. However, it may contain a small number of malignant cells, which may be overlooked during microscopic examination. In the present study, expression of MAGE-A3, -A4, -A6 and -A12 by hyperplastic leukoplakia indicated the existence of such a small number of malignant cells, as the lesion progressed to severe dysplasia and OSSC over time. Therefore, MAGE-A expression analysis can aid in the prediction of malignant transformation, and may prove beneficial if used as an additional parameter for cancer risk assessment, particularly for the long-term follow-up of patients with persistent oral lesions having clinically suspected manifestations. Our findings suggest that the expression of MAGE-A is capable of characterizing the malignant phenotype in leukoplakia, as well as its potential risk of developing malignancy. Thus, MAGE-A may be an additional reliable molecular diagnostic and prognostic marker for oral cancer development, and may help to differentiate physiological discrepancies from dysplastic ones. However, more carefully designed studies are needed to determine the expression profiles of dysplastic lesions that appear to have no or high potential for malignant transformation, in particular to clarify the role of such molecular biological methods as an additional tool for the cancer risk assessment of oral pre-malignant lesions.

In conclusion, the expression analysis of multiple MAGE-A genes may serve as a highly sensitive molecular diagnostic marker for the prediction of early malignant transformation in persistent pre-malignant oral lesions such as leukoplakia. This method might enable the timerly prediction of potential malignant changes, as well as improved treatment planning for the patient, thus decreasing the incidence of oral cancer.

\section{Acknowledgements}

This study was supported by the Johannes and Frieda Marohn Foundation and the ELAN grant from the University of Erlangen. The authors would also like to thank Ms. A. Krautheim-Zenk for valuable technical support.

\section{References}

1. Hunter KD, Parkinson EK and Harrison PR: Profiling early head and neck cancer. Nat Rev Cancer 5: 127-135, 2005.

2. Scheifele C and Reichart PA: Oral leukoplakia in manifest squamous epithelial carcinoma. A clinical prospective study of 101 patients. Mund Kiefer Gesichtschir 2: 326-330, 1998.

3. Jungbluth AA, Busam KJ, Kolb D, et al: Expression of MAGEantigens in normal tissues and cancer. Int J Cancer 85: 460-465, 2000.

4. Boon T, De Plaen E, Lurquin C, et al: Identification of tumour rejection antigens recognized by T lymphocytes. Cancer Surv 13: 23-37, 1992

5. Chomez P, De Backer O, Bertrand M, De Plaen E, Boon T and Lucas S: An overview of the MAGE gene family with the identification of all human members of the family. Cancer Res 61: 5544-5551, 2001.

6. De Plaen E, Arden K, Traversari C, et al: Structure, chromosomal localization and expression of 12 genes of the MAGE family. Immunogenetics 40: 360-369, 1994.

7. Van der Bruggen P, Traversari C, Chomez P, et al: A gene encoding an antigen recognized by cytolytic $\mathrm{T}$ lymphocytes on a human melanoma. Science 254: 1643-1647, 1991.

8. Mollaoglu N, Vairaktaris E, Nkenke E, Neukam FW and Ries J: Expression of MAGE-A12 in oral squamous cell carcinoma. Dis Markers 24: 27-32, 2008. 
9. Ries J, Schultze-Mosgau S, Neukam F, Diebel E and Wiltfang J: Investigation of the expression of melanoma antigen-encoding genes (MAGE-A1 to -A6) in oral squamous cell carcinomas to determine potential targets for gene-based cancer immunotherapy. Int J Oncol 26: 817-824, 2005.

10. Ries J, Vairaktaris E, Mollaoglu N, Wiltfang J, Neukam FW and Nkenke E: Expression of melanoma-associated antigens in oral squamous cell carcinoma. J Oral Pathol Med 37: 88-93, 2008

11. Eura M, Ogi K, Chikamatsu K, et al: Expression of the MAGE gene family in human head-and-neck squamous-cell carcinomas. Int J Cancer 64: 304-308, 1995.

12. Park JW, Kwon TK, Kim IH, et al: A new strategy for the diagnosis of MAGE-expressing cancers. J Immunol Methods 266: 79-86, 2002.

13. Parkin DM, Bray F, Ferlay J and Pisani P: Global cancer statistics, 2002. CA Cancer J Clin 55: 74-108, 2005.

14. Reibel J: Prognosis of oral pre-malignant lesions: significance of clinical, histopathological and molecular biological characteristics. Crit Rev Oral Biol Med 14: 47-62, 2003.

15. Warnakulasuriya S, Reibel J, Bouquot J and Dabelsteen E: Oral epithelial dysplasia classification systems: predictive value, utility, weaknesses and scope for improvement. J Oral Pathol Med 37: 127-133, 2008.

16. Ha PK, Benoit NE, Yochem R, et al: A transcriptional progression model for head and neck cancer. Clin Cancer Res 9: 3058-3064, 2003.
17. Ha PK, Chang SS, Glazer CA, Califano JA and Sidransky D: Molecular techniques and genetic alterations in head and neck cancer. Oral Oncol 45: 335-339, 2009.

18. Simpson AJ, Caballero OL, Jungbluth A, Chen YT and Old LJ: Cancer/testis antigens, gametogenesis and cancer. Nat Rev Cancer 5: 615-625, 2005 .

19. Robertson KD and Wolffe AP: DNA methylation in health and disease. Nat Rev Genet 1: 11-19, 2000.

20. Ehrlich M: DNA methylation in cancer: too much, but also too little. Oncogene 21: 5400-5413, 2002.

21. Clark SJ and Melki J: DNA methylation and gene silencing in cancer: which is the guilty party? Oncogene 21: 5380-5387, 2002.

22. De Smet C, De Backer O, Faraoni I, Lurquin C, Brasseur F and Boon T: The activation of human gene MAGE-1 in tumor cells is correlated with genome-wide demethylation. Proc Natl Acad Sci USA 93: 7149-7153, 1996

23. De Smet C, Lurquin C, Lethe B, Martelange V and Boon T: DNA methylation is the primary silencing mechanism for a set of germ line- and tumor-specific genes with a CpG-rich promoter. Mol Cell Biol 19: 7327-7335, 1999.

24. De Smet C, Loriot A and Boon T: Promoter-dependent mechanism leading to selective hypomethylation within the 5 ' region of gene MAGE-A1 in tumor cells. Mol Cell Biol 24: 4781-4790, 2004. 